\title{
NONLINEAR AND LINEAR ELASTIC IMPACT THEORY*
}

\author{
SHING-TUNG YAU $^{\dagger}$ AND WEN ZHANG ${ }^{\ddagger}$
}

\begin{abstract}
The elastic structure impact problems are studied in the paper. Not only the local impact regions are regarded as deformable, but also the whole structure is considered as a flexible one. At the local impact regions, the non-linear constitutive equation called Hertz law is used, while the deflection of the flexible structure is assumed to obey the linear Hooke's law. Both analytical and numerical studies have been done. For comparation, a simplified linear impact model is also introducted and studied in the paper. All the results show that the impact process has a foundamental pattern due to the local deformation at the impact regions and with some additional effects due to the influence of the structure flexibility.
\end{abstract}

1. Introduction. Impact process is a very complex mechanical phenomenon indeed. The main property is that the time process is very short $\left(10^{-3} \mathrm{sec}\right.$. order $)$ and the magnitude level of the impact force is very high. The simplest impact model is the rigid body impact model [3], in which, the time interval of impact process is assumed to be zero and the magnitude of the impact force is infinite, while the impulse remains limited. In this model, we only concern with some major integral quantities before and after the impaction and pay no attention on the impact process. Of course, in this model the local deformations at the two impact regions due to large impact force are also ignored.

The better model is to count in the elastic or plastic deformation at the local impact regions, while the other regions are still regarded as the rigid parts. This model can be called local deformation model. In this model, it is revealed that any impact process has a short time interval. The impact force varies from zero to a large but finite value and then fall down to zero again during the whole short impact interval. The constitutive equation of impact force and relative deformation between two impact bodies, called Hertz law, is not a linear relationship. So the impact phenomenon is essentially a non-linear mechanical problem.

If the impacted structure is so flexible that its lowest natural frequency has the same order of magnitude as, or even much lower than that of the impact frequency (the reciprocal of the impact period), the whole structure must be regarded as a deformable one during the impact process. This is called global deformation model. Although the structure deflection is still assumed to obey the Hooke's law, the local deformation at the impact regions remains non-linear.

In this paper we study the third model in detail. The general non-linear equation of motion is established. It is an integral equation of Voltera type. The general analytical solution is deduced by a perturbation method. The numerical computation is done by difference method. For comparision, a simplified linear impact constitutive equation is used to linearize the problem. In this case, the problem is reduced to a

\footnotetext{
*Received December 17, 1999.

†Department of Mathematics, Harvard University, Cambridge, MA 02138, U.S.A. and The Institute of Mathematical Sciences \& Department of Mathematics, CUHK, Hong Kong (yau@ MATH.HARVARD.EDU).

${ }_{\ddagger}$ Department of Applied Mechanics, Fudan University, Shanghai, China. Research supported by NFS/DARPA grant DMA 87-11394.
} 
initial problem of vibration which can be solved analytically.

All the results obtained in the paper show that the major impact pattern of the global deformation model is not very far from the pattern of the local deformation model which can be called as fundamental pattern. And the additional effect of structure flexibility is mainly due to these normal vibration models of the structure whose natural frequencies are not very far from the impact frequency of the local deformation model.

2. Forced Motion Solution of Elastic System. The forced vibration solution for multiple degree of freedom linear system is reviewed here briefly. The context described here is only those necessary fundamental material which is useful for developing the following nonlinear impact theory of elastic system.

The natural dynamics of an elastic system with $n$ degrees of freedom is described by $\omega_{i}$ and $\phi_{i}(i=1, \cdots, n)$ of the following eigenvalue problem:

$$
K \phi_{i}=\omega_{i}^{2} M \phi_{i}
$$

or

$$
K \Phi=M \Phi \Lambda
$$

where

$$
\Lambda=\left[\begin{array}{lll}
\ddots & & \\
& \omega_{i}^{2} & \\
& & \ddots
\end{array}\right]
$$

and $\Phi$ satisfies the following orthogonal identities:

$$
\begin{gathered}
\Phi^{T} M \Phi=\left[\begin{array}{lll}
\ddots & & \\
& m_{i} & \\
& & \ddots
\end{array}\right], \\
\Phi^{T} K \Phi=\left[\begin{array}{llll}
\ddots & & \\
& k_{i} & \\
& & \ddots
\end{array}\right]=\left[\begin{array}{llll}
\ddots & & \\
& m_{i} \omega_{i}^{2} & \\
& & \ddots
\end{array}\right] .
\end{gathered}
$$

All the $\phi_{i}(i=1, \cdots, n)$ are normalized by $\left|\phi_{i}\right|_{\max }=1$. Here $m_{i}$ is called the $i$-th model mass.

If a set of external forces, $f_{1}(t), f_{2}(t), \cdots, f_{n}(t)$, act on the system, the equation of motion is given by

$$
M \ddot{X}+K X=F(t)
$$


where $F^{T}(t)=\left[f_{1}(t), f_{2}(t), \cdots, f_{n}(t)\right] . f_{i}(t)$ is the force acting on the $i$-th node.

Now let

$$
X(t)=\sum_{i=1}^{n} \phi_{i} \cdot \xi_{i}(t)=\Phi \Xi
$$

where

$$
\Xi=\left[\xi_{1}, \xi_{2}, \cdots, \xi_{n}\right]^{T}
$$

Substituting (8) into (7) and multiplying by $\Phi^{T}$, we get

$$
\Phi^{T} M \Phi \ddot{\xi}+\Phi^{T} K \Phi \xi=R(t)
$$

or

$$
m_{i} \ddot{\xi}_{i}+k_{i} \xi_{i}=r_{i}(t), \quad(i=1, \cdots, n)
$$

where

$$
R(t)=\left[r_{1}(t), r_{2}(t), \cdots, r_{n}(t)\right]^{T}=\Phi^{T} F
$$

The solution of (11) with the initial conditions $\xi_{i}(0)=0$ and $\xi_{i}^{\prime}(0)=0$ is given by

$$
\xi_{i}=\frac{1}{m_{i} \omega_{i}} \int_{0}^{t} r_{i}(r) \cdot \sin \omega_{i}(t-\tau) d \tau, \quad(i=1, \cdots, n)
$$

or

$$
\Xi=\int_{0}^{t}\left(\begin{array}{lll}
\ddots & & \\
& \frac{\sin \omega_{i}(t-\tau)}{m_{i} \omega_{i}} & \\
& & \ddots
\end{array}\right) \Phi^{T} F(\tau) d \tau
$$

Substituting (14) into (8), we get the general solution of forced motion as follows:

$$
X=\Phi \int_{0}^{t}\left(\begin{array}{lll}
\ddots & & \\
& \frac{\sin \omega_{i}(t-\tau)}{m_{i} \omega_{i}} & \\
& & \ddots
\end{array}\right) \Phi^{T} F(\tau) d \tau
$$

If there is only a single force $f(t)$ acting on the system, say, at the $j$-th node, then $F(t)=[0, \cdots, 0, f(t), 0, \cdots, 0]^{T}$. The elastic deflection $x_{0}$ at the $j$-th node, according to $(15)$, is given by

$$
x_{0}=\sum_{i=1}^{n} \frac{\phi_{i 0}^{2}}{m_{i} \omega_{i}} \int_{0}^{t} f(\tau) \cdot \sin \omega_{i}(t-\tau) d \tau
$$

where

$$
\phi_{i 0}=\left.\phi_{i}\right|_{\text {at }} \text { the } j \text {-th node }
$$


3. Hertz Law for Elastic Impact Between Two Bodies. For the case of elastic impact between two bodies, the constitutive equation, namely, the Hertz law [1] is given by

$$
f(t)=\alpha \delta^{\frac{3}{2}}(t)
$$

where $\delta(t)$ is the relative displacement between the two impact points, $f(t)$ is the contact force during the impact time interval. Equation (18) expresses a nonlinear relation between the impact force and the relative displacement. The coefficient $\alpha$ is determined by the following formula [2]:

$$
\alpha=\frac{4}{3\left(\frac{1-\nu_{1}^{2}}{E_{1}}+\frac{1-v_{2}^{2}}{E_{2}}\right) \sqrt{k_{1}+k_{2}}}
$$

where $k_{i}(i=1,2)$ are the average curvatures of the two local surfaces at the two impact points respectively. $E_{i}$ and $\nu_{i}(i=1,2)$ are the Young's moduli and Poission's ratios of the two impact materials respectively. If the two local impact surfaces are a ball surface and a plane respectively, $k_{1}=1 / R_{1}$ and $k_{2}=0$, then

$$
\alpha=\frac{4}{3} \cdot \frac{\sqrt{R_{1}}}{\frac{1-\nu_{1}^{2}}{E_{1}}+\frac{1-\nu_{2}^{2}}{E-2}}
$$

where $R_{1}$ is the radius of the local ball surface.

4. Ball-Rigid Plate Impact. First, let us discuss the simplest case that a ball impacts towards a rigid plate with an initial velocity $v_{0}$. During the impact period, $0 \leq t \leq t_{m}$, the displacement of the ball is

$$
x(t)=v_{0} t-\frac{1}{m_{0}} \int_{0}^{t} d t_{1} \int_{0}^{t_{1}} f(\tau) d \tau, \quad\left(0 \leq t \leq t_{m}\right)
$$

where $f(t)$ is the impact force, $m_{0}$ is the mass of the ball.

Note that (21) is just the relative displacement $\delta(t)$ between the ball and the wall, because the wall is rigidly fixed and cannot have any elastic displacement excited by the impact force $f(t)$. So we have

$$
\delta(t)=v_{0} t-\frac{1}{m_{0}} \int_{0}^{t} d t_{1} \int_{0}^{t_{1}} f(\tau) d \tau, \quad\left(0 \leq t \leq t_{m}\right)
$$

Substituting the Hertz law (18) into (22), we get

$$
v_{0} t-\frac{\alpha}{m_{0}} \int_{0}^{t} d t_{1} \int_{0}^{t_{1}} \delta^{\frac{3}{2}}(\tau) d \tau=\delta(t), \quad\left(0 \leq t \leq t_{m}\right)
$$

This is the derived non-linear impact equation. After twice derivatives of (23), the following non-linear differential equation of motion for $\delta(t)$ is obtained:

$$
m_{o} \ddot{\delta}+\alpha \delta^{\frac{3}{2}}=0
$$

with the following initial conditions:

$$
\delta(0)=0, \quad \dot{\delta}(t)=v_{0}
$$


The equation (24) can be easily integrated as

$$
\dot{\delta}^{2}-v_{0}^{2}+\frac{4}{5} \frac{\alpha}{m_{0}} \delta^{\frac{5}{2}}=0
$$

or

$$
\dot{\delta}= \pm v_{0} \sqrt{1-\left(\frac{\delta}{\delta_{m}}\right)^{\frac{5}{2}}}
$$

where

$$
\delta_{m}\left(\frac{5}{4} \frac{m_{0} v_{0}^{2}}{\alpha}\right)^{\frac{2}{5}}=\left[\frac{15}{16} \frac{m_{0} v_{0}^{2}}{\sqrt{R_{1}}}\left(\frac{1-\nu_{1}^{2}}{E-1}+\frac{1-\nu_{2}^{2}}{E_{2}}\right)\right]^{\frac{2}{5}}
$$

(27) can be further integrated to obtain

$$
\int_{0}^{\frac{\delta}{\delta_{m}}} \frac{d x}{\sqrt{1-x^{\frac{5}{2}}}}= \begin{cases}\frac{v_{0} t}{\delta_{m}} & 0 \leq t \leq c \\ \frac{v_{0} t}{\delta_{m}}(2 c-t) & c \leq t \leq 2 c\end{cases}
$$

(29) is the obtained complete solution of $\delta(t)$, which is sketched out in Fig. 1 by the solid line, where

$$
c=\int_{0}^{1} \frac{d t}{\sqrt{1-x^{\frac{5}{2}}}} \approx 1.4716
$$

The impact interval, $t_{m}$, then can be obtained as

$$
t_{m}=2 c \frac{\delta_{m}}{v_{0}} \approx 2.9432 \frac{\delta_{0}}{v_{0}} \approx 3 \frac{\delta_{0}}{v_{0}}
$$

As the conclusion of this section, we list some exact results obtained above for ball-rigid plate impact:

1. The maximum relative displacement:

$$
\delta_{m}=\left(\frac{5}{4} \frac{m_{0} v_{0}^{2}}{\alpha}\right)^{\frac{2}{5}}
$$

2. The maximum amplitude of the impact force:

$$
f_{m}=\alpha \delta_{m}^{\frac{3}{2}}=\alpha\left(\frac{5}{4} \frac{m_{0} v_{0}^{2}}{\alpha}\right)^{\frac{3}{5}}
$$

3. The impact stiffness coefficient:

$$
k_{m}=\frac{f_{m}}{\delta_{m}}=\alpha \delta_{m}^{\frac{1}{2}}
$$

4. The impact flexibility coefficient:

$$
\alpha_{m}=\frac{1}{k_{m}}=\frac{\delta_{m}}{f_{m}}
$$

5. The impact period:

$$
t_{m}=2 c \frac{\delta_{m}}{v_{0}} \approx \frac{3 \delta_{m}}{v_{0}}
$$

6. The impact frequency:

$$
\omega_{0}=\frac{v_{0}}{\delta_{m}} \approx \frac{\pi}{3} \frac{v_{0}}{\delta_{m}} \approx \frac{\pi}{t_{m}}
$$




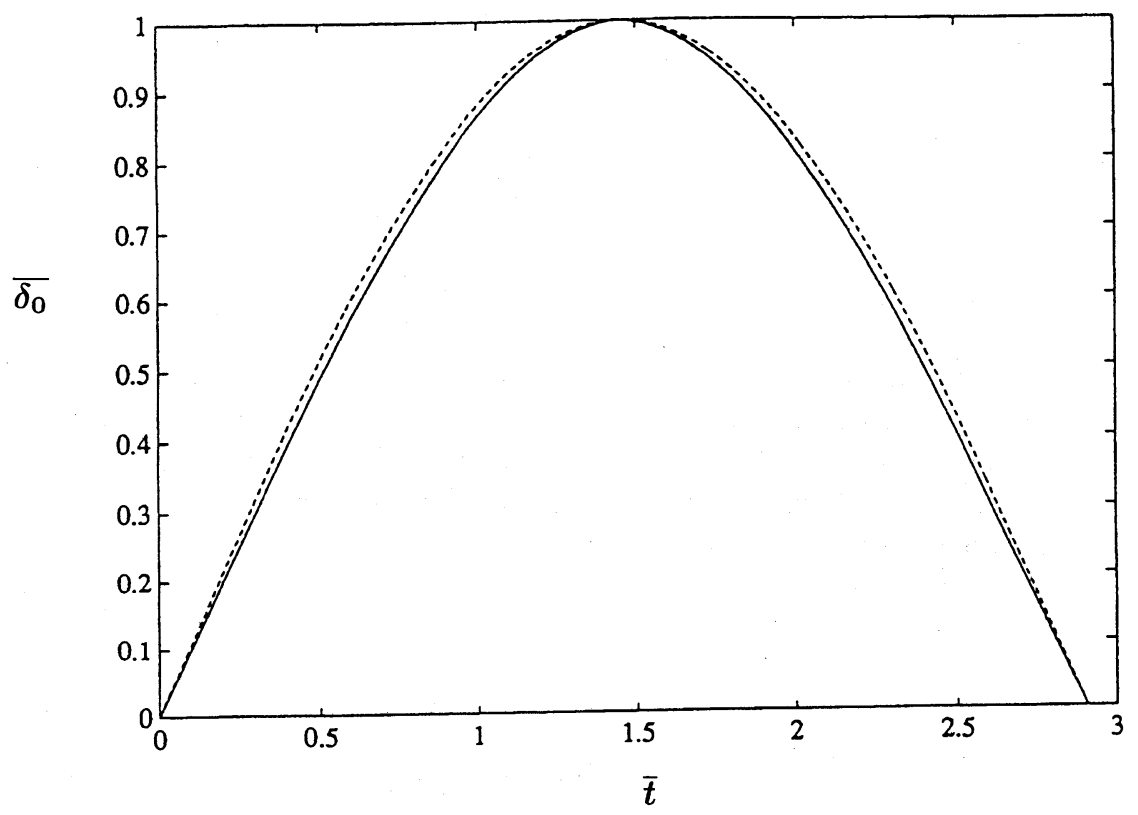

FIG. 1.

5. Impact Between Ball and Elastic Structure. Indeed the most common impact case is that a ball impacts to an elastic structure. Let the mass and the initial impact velocity of the ball be still denoted by $m_{0}$ and $v_{0}$ respectively. During the impact period $\left(0 \leq t \leq t_{m}\right)$, the absolute displacement of the ball is

$$
x_{1}(t)=v_{0} t-\frac{1}{m_{0}} \int_{0}^{t} d t_{1} \int_{0}^{t_{1}} f(\tau) d \tau, \quad\left(0 \leq t \leq t_{m}\right),
$$

whereas the absolute displacement of the impact point on the elastic structure, according to (16), is

$$
x_{2}(t)=\sum_{i=1}^{n} \frac{\phi_{i 0}^{2}}{m_{i} \omega_{i}} \int_{0}^{t} f(\tau) \cdot \sin \omega_{i}(t-\tau) d \tau, \quad\left(0 \leq t \leq t_{m}\right)
$$

The relative displacement of the two impact points is

$$
\delta=x_{1}-x_{2}
$$

or

$$
x_{1}=x_{2}+\delta
$$

Substituting (38) and (39) into (41), we come to the following equation:

$$
\begin{gathered}
v_{0} t-\frac{1}{m_{0}} \int_{0}^{t} d t_{1} \int_{0}^{t_{1}} f(\tau) d \tau=\sum_{i=1}^{n} \frac{\phi_{i 0}^{2}}{m_{i} \omega_{i}} \int_{0}^{t} f(\tau) \sin \omega_{i}(t-\tau) d \tau+\delta(t) \\
\left(0 \leq t \leq t_{m}\right)
\end{gathered}
$$


or

$$
\begin{gathered}
v_{0} t-\frac{\alpha}{m_{0}} \int_{1}^{t} d t_{1} \int_{0}^{t_{1}} \delta^{\frac{3}{2}}(\tau) d \tau=\alpha \sum_{i=1}^{n} \frac{\phi_{i 0}^{2}}{m_{i} \omega_{i}} \int_{0}^{t} \quad \delta^{\frac{3}{2}}(\tau) \sin \omega_{i}(t-\tau) d \tau+\delta(t) \\
\left(0 \leq t \leq t_{m}\right)
\end{gathered}
$$

Equation (42) is the nonlinear impact equation deduced here, from which $\delta(t)$ can be solved. And the impact period $t_{m}$ can be obtained by following condition:

$$
\delta\left(t_{m}\right)=0
$$

It is difficult to find out analytical solution of (42). Usually it should be solved numerically step by step with time $t$ by a difference method. For this reason, it is much convenient to alter (42) into a non-dimensional form. Taking the characteristic quantities of (32)-(37) for ball-rigid plate impact and the ball mass $m_{0}$ as the scale quantities, all the non-dimensional quantities can be defined as follows:

$$
\begin{aligned}
\bar{f}(t) & =\frac{f(t)}{f_{m}} \\
\bar{\delta}(t) & =\frac{\delta(t)}{\delta_{m}} \\
\bar{t} & =\omega_{0} t \\
\bar{\tau} & =\omega_{0} \tau \\
\bar{\omega}_{i} & =\frac{\omega_{i}}{\omega_{0}} \\
\bar{m}_{i} & =\frac{m_{i}}{m_{0}}
\end{aligned}
$$

Substituting all above quantities into (42) and (18), the general non-dimensional impact equation and Hertz law are written as

$$
\begin{array}{cc}
\bar{t}-\frac{5}{4} \int_{0}^{\bar{t}} d \bar{t}_{1} \int_{0}^{\bar{t}_{1}} \bar{\delta}^{\frac{3}{2}}(\bar{\tau}) d \bar{\tau}=\frac{5}{4} \sum_{i=1}^{n} \frac{\phi_{i 0}^{2}}{\bar{m}_{i} \bar{\omega}_{i}} \int_{0}^{\bar{t}} \bar{\delta}^{\frac{3}{2}}(\bar{\tau}) \sin \bar{\omega}_{i}(\bar{t}-\bar{\tau}) d \bar{\tau}+\bar{\delta}(\bar{t}) \\
\left(0 \leq \bar{t} \leq \bar{t}_{m}\right) \\
\bar{f}(\bar{t})=\bar{\delta}^{\frac{3}{2}}(\bar{t}),
\end{array}
$$

where $\bar{t}_{m}$ satisfies

$$
\bar{\delta}\left(\bar{t}_{m}\right)=0
$$

6. Linear Theory. By virtue of the approximate simple linear theory, we can inspect some important impact properties analytically. It is also helpful to numerically solve the non-linear equation (44) as a comparison.

6.1. Linear Impact Constitutive Equation. In the linear impact theory, the Hertz law (18) should be replaced by the following linear approximation

$$
f(t)=k_{0} \cdot \delta(t)
$$


where $k_{0}$ is an equivalent stiffness coefficient, which can be determined by the following strain energy balance consideration:

$$
\frac{k_{0}}{2} \delta_{m}^{2}=\int_{0}^{\delta_{m}} \alpha \delta^{\frac{3}{2}} d \delta=\alpha \cdot \frac{2}{5} \delta_{m}^{\frac{5}{2}}
$$

i.e.,

$$
k_{0}=\frac{4}{5} \alpha \delta_{m}^{\frac{1}{2}}=\left(\frac{4}{5}\right)^{\frac{4}{5}} \alpha^{\frac{4}{5}}\left(m_{0} v_{0}^{2}\right)^{\frac{1}{5}}
$$

6.2. Equation of Motion. Substituting (47) into (42), the impact equation is reduced to

$$
v_{0} t-\frac{k_{0}}{m_{0}} \int_{0}^{t} d t_{1} \int_{0}^{t_{1}} \delta(\tau) d \tau=\sum_{i=1}^{n} \frac{\phi_{i 0}^{2} k_{0}}{m_{i} \omega_{i}} \int_{0}^{t} \delta(\tau) \sin \omega_{i}(t-\tau) d \tau+\delta(t) .
$$

Letting

$$
q_{i}(t)=\frac{\phi_{i 0} k_{0}}{m_{i} \omega_{i}} \int_{0}^{t} \delta(\tau) \sin \omega_{i}(t-\tau) d \tau
$$

(50) then can be written as

$$
v_{0} t-\frac{k_{0}}{m_{0}} \int_{0}^{t} d t_{1} \int_{0}^{t_{1}} \delta(\tau) d \tau=\sum_{i=1}^{n} \phi_{i 0} q_{i}(t)+\delta(t)
$$

By differentiating (52) and (51) twice respectively with respect to time $t$, we get the following equivalent set of differential equations of motion

$$
-\omega_{0}^{2}\left(\begin{array}{lll}
\ddots & & \\
\ddots & \frac{m_{0}}{m_{j}} & \\
& & \ddots
\end{array}\right) \Phi_{0}^{T} \delta+\ddot{q}+\left(\begin{array}{lll}
\ddots & & \\
& \omega_{j}^{2} & \\
& & \ddots
\end{array}\right) \boldsymbol{q}=0
$$

where

$$
\begin{aligned}
\Phi_{0} & =\left[\phi_{10}, \phi_{20}, \cdots, \phi_{n 0}\right] \\
\boldsymbol{q} & =\left[q_{1}, q_{2}, \cdots, q_{n}\right]^{T} \\
\omega_{0} & =\sqrt{\frac{k_{0}}{m_{0}}}
\end{aligned}
$$

It can be justified that $\omega_{0}=\sqrt{\frac{k_{0}}{m_{0}}}$ is equal to the impact frequency (37) defined in section 4 .

It should be pointed out that from (39) and (51) we have

$$
x_{2}=\sum_{i=1}^{2} \phi_{i 0} \cdot q_{i}=\Phi_{0}^{T} \boldsymbol{q}
$$


So $q=\left[q_{1}, q_{2}, \cdots, q_{n}\right]^{T}$ is the set of $n$ normal mode coordinates of the elastic structure. Thus the set of equations (53) can be obtained alternatively by Lagrange procedure with the following kinetic and potential energy expressions of the linear impact system:

$$
\begin{aligned}
& T=\frac{1}{2} m_{0} \dot{x}_{1}^{2}+\frac{1}{2} \dot{x}_{2}^{2}=\frac{1}{2} m_{0}\left(\dot{\delta}+\Phi_{0} \dot{\boldsymbol{q}}\right)^{2}+\frac{1}{2} \dot{\boldsymbol{q}}^{T}\left[m_{i}\right] \dot{\boldsymbol{q}} \\
& V=\frac{1}{2} k_{0} \delta^{2}+\frac{1}{2} \boldsymbol{q}^{T}\left(\begin{array}{lll}
\ddots & & \\
& m_{i} \omega_{i}^{2} & \\
& & \ddots
\end{array}\right) q .
\end{aligned}
$$

6.3. Solutions of the Linear Equations. Letting $\delta=\tilde{\delta} e^{i p t}$ and $\boldsymbol{q}=\tilde{\boldsymbol{q}} e^{i p t}$ and substituting them into (53), we get the eigenvalue problem of the linear system:

$$
-\omega_{0}^{2}\left(\begin{array}{lll}
\ddots & & \\
& \frac{m_{0}}{m_{j}} & \\
& & \ddots
\end{array}\right) \Phi_{0}^{T} \tilde{\delta}+\left(\begin{array}{lll}
\ddots & & \\
& \omega_{j}^{2}-p^{2} & \\
& & \ddots
\end{array}\right) \tilde{\delta}-p^{2} \Phi_{0} \tilde{\boldsymbol{q}}=0,0
$$

Form the second equation of (60), we have

$$
\tilde{\boldsymbol{q}}=\omega_{0}^{2}\left(\begin{array}{lll}
\ddots & & \\
& \frac{m 0}{m_{j}\left(\omega_{j}^{2}-p^{2}\right)} & \\
& & \ddots
\end{array}\right) \Phi_{0}^{T} \tilde{\delta}
$$

Substituting it into the first equation of (60), we get

$$
\omega_{0}^{2}-p^{2}-p^{2} \omega_{0}^{2} \Phi_{0}\left(\begin{array}{lll}
\ddots & & \\
& \frac{m_{0}}{m_{j}\left(\omega_{j}^{2}-p^{2}\right)} & \\
& & \ddots
\end{array}\right) \Phi_{0}^{T}=0
$$

This is the characteristic equation of the eigenvalue problem (60)

Once all the $n+1$ eigenvalues $p_{i}(i=1,2, \cdots, n+1)$ are evaluated from $(62)$, the corresponding eigenvectors $\tilde{Q}_{i}=\left[\tilde{\delta}: \tilde{q}^{T}\right]_{i}^{T}(i=1,2, \cdots, n+1)$ can be given from (61) as

$$
\left.\tilde{\boldsymbol{Q}}_{i}=\left[\begin{array}{c}
\tilde{\delta} \\
\cdots \\
\tilde{\boldsymbol{q}}
\end{array}\right]_{i}=\left[\begin{array}{ccc} 
& \\
& \ldots \ldots & \\
\ddots & & \\
& \frac{\omega_{0}^{2} m_{0}}{m_{j}\left(\omega_{j}^{2}-p_{i}^{2}\right)} & \\
& & \ddots
\end{array}\right) \Phi_{0}^{T}\right]
$$

or alternatively

$$
\left\{\begin{array}{l}
\tilde{\delta}_{i}=1 \\
\tilde{q}_{j i}=\frac{\omega_{0}^{2} m_{0}}{m_{j}\left(\omega_{j}^{2}-p_{i}^{2}\right)} \phi_{j 0}, \quad(j=1,2, \cdots, n) \\
\quad(i=1,2, \cdots, n+1) .
\end{array}\right.
$$


6.4. Impact Solution. Now the impact problem of linear model is classified into the free vibration problem with the following initial conditions:

$$
\begin{aligned}
& \delta(0)=0, \boldsymbol{q}=0 \\
& \dot{\delta}(0)=0, \dot{\boldsymbol{q}}(0)=0
\end{aligned}
$$

The solution satisfying (65) has the following general form:

$$
\left[\begin{array}{c}
\delta(t) \\
\cdots \\
\boldsymbol{q}(t)
\end{array}\right]=\left[\tilde{Q}_{1}, \tilde{Q}_{2}, \cdots, \tilde{Q}_{n+1}\right] \cdot\left[\begin{array}{c}
a_{1} \sin p_{1} t \\
a_{2} \sin p_{2} t \\
\vdots \\
a_{n+1} \sin p_{n+1} t
\end{array}\right]
$$

where coefficients $a_{i}(i=1,2, \cdots, n+1)$ in (67) should be determined by the initial velocity conditions (66), i.e.,

$$
\begin{gathered}
\sum_{i=1}^{n+1} a_{i} p_{i}=0 \\
\sum_{i=1}^{n+1} \frac{a_{i} p_{i}}{m_{j}\left(\omega_{j}^{2}-p_{i}^{2}\right)}=0 \quad(j=1,2, \cdots, n) .
\end{gathered}
$$

After $a_{i}(i=1,2, \cdots, n+1)$ have been determined from (68) and (69), $\delta(t)$ and $\boldsymbol{q}(t)$ are then obtained from (67). The linear impact problem is thus completely solved.

6.5. Perturbation Solution. It is difficult to get an explicit solution of $a_{i}$ from (68) and (69). However, $m_{0} / m_{j}(j=1, \cdots, n)$ are usually small comparing with 1. We can pursue a perturbation solution of $a_{i}$ from (68) and (69).

First, if $m_{0} / m_{j}=0,(j=1, \cdots, n)$, the $i$-th eigensolution can be obtained directly from (60) and that

$$
\begin{aligned}
p_{i}=\omega_{i}, \tilde{Q}_{i}=\left\{\begin{array}{l}
\tilde{\delta}_{i}=1 \\
\left(\tilde{q}_{j}\right)_{i}=0, \quad(j=1, \cdots, n, j \neq i) \\
\left(\tilde{q}_{i}\right)_{i}=\frac{\omega_{0}^{2}-\omega_{i}^{2}}{\omega_{i}^{2} \phi_{i 0}} \\
(i=1, \cdots, n)
\end{array}\right.
\end{aligned}
$$

and

$$
p_{n+1}=\omega_{0}, \tilde{Q}_{n+1}=\left\{\begin{array}{l}
\tilde{\delta}_{n+1}=1 \\
\left(\tilde{q}_{j}\right)_{n+1}=0, \quad(j=1, \cdots, n)
\end{array}\right.
$$

Substituting (70) and (71) into (68) and (69), we get

$$
a_{1}=\cdots=a_{n}=0, a_{n+1}=\frac{v_{0}}{\omega_{0}} .
$$

And from (67), we have

$$
\left\{\begin{array}{l}
\delta(t)=\frac{v_{0}}{\omega_{0}} \sin \omega_{0} t \\
\boldsymbol{q}(t)=\mathbf{0}
\end{array}\right.
$$


It is obvious that (73) is the ball-rigid body impact solution for linear impact model.

Now let the value of $m_{0} / m_{j}(j=1, \cdots, n)$ are all small

$$
\frac{m_{0}}{m_{j}}=\epsilon \bar{m}_{j},(j=1, \cdots, n)
$$

where $\epsilon$ is a small characteristic quantity. Taking (70) and (71) as the zero order approximate solution, then consider the following perturbation solution Eq. (60):

$$
\left\{\begin{array}{l}
p_{i}^{2}=\omega_{i}^{2}+\epsilon \bar{p}_{i}^{2} \\
\left(\tilde{q}_{i}\right)_{i}=\frac{\omega_{0}^{2}-\omega_{i}^{2}}{\omega_{i}^{2} \phi_{i 0}}+\epsilon\left(\bar{q}_{i}\right)_{i} \\
\left(\tilde{q}_{j}\right)_{i}=\epsilon\left(\bar{q}_{j}\right)_{i}
\end{array}\right.
$$

and

$$
\left\{\begin{array}{l}
p_{n+1}^{2}=\omega_{0}^{2}+\epsilon \bar{p}_{n+1}^{2} \\
\left(q_{j}\right)_{n+1}=\epsilon\left(\bar{q}_{j}\right)_{n+1}
\end{array},\right.
$$

where $\epsilon \bar{p}_{i}^{2}, \epsilon\left(\bar{q}_{i}\right)_{i}, \epsilon\left(\bar{q}_{j}\right)_{i}, \epsilon \bar{p}_{n+1}^{2}$, and $\epsilon\left(\bar{q}_{j}\right)_{n+1}$ are first order perturbation terms which are undetermined.

Now substituting (75) and (76) into (60), and reserving only all the first order terms, we obtain the following perturbation solutions:

$$
\begin{aligned}
p_{i}^{2} & =\omega_{0}^{2}+\frac{m_{0}}{m_{i}} \cdot \frac{\omega_{0}^{2} \omega_{i}^{2} \phi_{i 0}^{2}}{\omega_{i}^{2}-\omega_{0}^{2}} \\
\left(\tilde{q}_{i}\right)_{i} & =\frac{\omega_{0}^{2}-\omega_{i}^{2}}{\omega_{i}^{2} \phi_{i 0}}+\frac{m_{0}}{m_{i}} \cdot \frac{\omega_{0}^{4} \phi_{i 0}}{\omega_{i}^{2}\left(\omega_{0}^{2}-\omega_{i}^{2}\right)}+\frac{\omega_{0}^{2}}{\phi_{i 0}} \sum_{\substack{j=i \\
j \neq 1}}^{n} \frac{m_{0}}{m_{j}} \cdot \frac{\phi_{i 0}^{2}}{\omega_{i}^{2}-\omega_{j}^{2}} \\
\left(\tilde{q}_{j}\right) i & =\frac{m_{0}}{m_{j}} \cdot \frac{\omega_{0}^{2} \phi_{i 0}}{\omega_{j}^{2}-\omega_{i}^{2}}(i=1, \cdots, n)
\end{aligned}
$$

and

$$
\begin{aligned}
P_{n+1}^{2} & =\omega_{0}^{2}-\omega_{0}^{4} \sum_{j=1}^{n} \frac{m_{0}}{m_{j}} \frac{\phi_{i 0}^{2}}{\omega_{j}^{2}-\omega_{0}^{2}} \\
\left(q_{i}\right)_{n+1} & =\omega_{0}^{2} \frac{m_{0}}{m_{j}} \frac{\phi_{i 0}}{\omega_{j}^{2}-\omega_{0}^{2}},(j=1, \cdots, n) .
\end{aligned}
$$

Now consider the perturbation solutions of (68) and (69). Assuming

$$
\begin{cases}a_{i} & =\epsilon \bar{a}_{i},(j=1, \cdots, n) \\ a_{n+1} & =\frac{v_{0}}{\omega_{0}}+\epsilon \bar{a}_{n+1}\end{cases}
$$

Substituting (79), (77) and (78) into (68) and (69), we have

$$
\left\{\begin{array}{l}
\epsilon \bar{a}_{i}=\frac{m_{0}}{m_{i}} \cdot \frac{v_{0} \omega_{0}^{2} \omega_{i} \phi_{i 0}^{2}}{\left(\omega_{i}^{2}-\omega_{0}^{2}\right)^{2}},(i=1, \cdots, n) \\
\epsilon \bar{a}_{n+1}=-\sum_{i=1}^{n} \frac{m_{0}}{m_{i}} \cdot \frac{v_{0} \omega_{0}\left(\omega_{i}^{2}+\omega_{0}^{2}\right) \phi_{i 0}^{2}}{2\left(\omega_{i}^{2}-\omega_{0}^{2}\right)^{2}}
\end{array}\right.
$$


At last, from (67) we get the first order solution of the relative displacement

$$
\begin{aligned}
\delta(t)= & \sum_{i=1}^{n+1} a_{i} \sin p_{i} t \\
= & \frac{v_{0}}{\omega_{0}} \sin \left(\omega_{0}-\frac{1}{2} \omega_{0}^{3} \sum_{i=1}^{n} \frac{m_{0}}{m_{i}} \frac{\phi_{i 0}^{2}}{\omega_{i}^{2}-\omega_{0}^{2}}\right) t \\
& +\sum_{i=1}^{n} \frac{m_{0}}{m_{i}} \frac{v_{0} \omega_{0}^{2} \omega_{i} \phi_{i 0}^{2}}{\left(\omega_{i}^{2}-\omega_{0}^{2}\right)^{2}} \sin \omega_{i} t-\left(\sum_{i=1}^{n} \frac{m_{0}}{m_{i}} \frac{v_{0} \omega_{0}\left(\omega_{i}^{2}+\omega_{0}^{2}\right) \phi_{i 0}^{2}}{2\left(\omega_{i}^{2}-\omega_{0}^{2}\right)^{2}}\right) \sin \omega_{0} t
\end{aligned}
$$

From above solution (81) we come to the following conclusions:

1. The major pattern of ball-elastic structure impact is not very far from that of the corresponding ball-rigid body impaction, which is expressed by $\delta(t)=$ $\frac{v_{0}}{m_{0}} \sin \omega_{0} t$. This fundamental pattern is purely due to the local deformation at the impact regions.

2. The additional effect of the structure elasticity is mainly due to these normal modes whose natural frequencies $\omega_{i}$ are not very far from the impact frequency $\omega_{0}$.

7. Perturbation Solution for Non-linear Impact Equation. The perturbation method also can be used to solve the non-linear impact equation (44). For the sake of convenience, differentiate eq. (44) twice with respect to time $\bar{t}$ and then integrate it once with respect to space $\bar{\delta}$. The result is

$$
\dot{\bar{\delta}}^{2}-1+\bar{\delta}^{\frac{5}{2}}=-\frac{5}{2} \sum_{j=1}^{n} \frac{m_{0} \phi_{i 0}^{2}}{m_{j}} \int_{0}^{\bar{t}} \dot{\bar{\delta}}(\bar{\xi})\left(\frac{d}{d \bar{\xi}} \int_{0}^{\bar{\xi}} \bar{\delta}^{\frac{3}{2}}(\bar{\tau}) \cdot \cos \bar{\omega}_{j}(\bar{\xi}-\bar{\tau}) d \bar{\tau}\right) d \bar{\xi}
$$

When $m_{0} / m_{j}=0,(j=1, \cdots, n)$, the zero order solution $\bar{\delta}_{0}(t)$ is just the solution (29) for ball-rigid body model. Its non-dimensional form is

$$
\int_{0}^{\bar{\delta}_{0}} \frac{d x}{\sqrt{1-x^{\frac{5}{2}}}}=\bar{t}
$$

The curve (83) is shown in Fig. 1 by the solid line, which can be approximated by a half period sine curve:

$$
\bar{\delta}_{0}(\bar{t})=\sin e_{0} \bar{t}
$$

where

$$
e_{0}=\frac{\pi}{2 c} \approx 1.0674
$$

and $c$ is defined by (30). The curve (84) is also shown in Fig. 1 by the line of dashes.

Now let us consider the perturbation solution of (82). Assuming $m_{0} / m_{j}(j=$ $1, \cdots, n)$ are small quantities just as before, set

$$
\bar{\delta}(\bar{t})=\bar{\delta}_{0}(\bar{t})+\epsilon \bar{\delta}_{1}(\bar{t})
$$


where $\epsilon \bar{\delta}_{1}(\bar{t})$ is an unknown first order perturbation term. Substituting (86) into (82), reserving the first order terms only, we get

$$
\dot{\delta}_{0} \dot{\bar{\delta}}_{1}+\frac{5}{4} \bar{\delta}_{0}^{\frac{3}{2}} \bar{\delta}_{1}=-\frac{5}{4} \int_{0}^{\bar{t}} \dot{\delta}_{0}(\bar{\xi})\left(\frac{d}{d \bar{\xi}} \int_{0}^{\bar{\xi}} \bar{\delta}_{0}^{\frac{3}{2}}(\bar{\tau}) \sum_{j=1}^{n} \frac{m_{0} \phi_{i 0}^{2}}{m_{j}} \cos \bar{\omega}_{j}(\bar{\xi}-\bar{\tau}) d \bar{\tau}\right) d \bar{\xi}
$$

From (83) we further have

$$
\ddot{\bar{\delta}}_{0}=-\frac{5}{4} \bar{\delta}_{0}^{\frac{3}{2}}
$$

Substituting above equation into (87), we get

$$
\dot{\bar{\delta}}_{0}^{2} \frac{d}{d \bar{t}}\left(\frac{\bar{\delta}_{1}}{\dot{\bar{\delta}}_{0}}\right)=\int_{0}^{\bar{t}} \dot{\bar{\delta}}_{0}(\bar{\xi})\left(\frac{d}{d \bar{\xi}} \int_{0}^{\bar{\xi}} \ddot{\bar{\delta}}_{0}(\bar{\tau}) \sum_{j=1}^{n} \frac{m_{0} \phi_{i 0}^{2}}{m_{j}} \cos \bar{\omega}_{j}(\bar{\xi}-\bar{\tau}) d \bar{\tau}\right) d \bar{\xi}
$$

which can be further integrated out as

$$
\bar{\delta}_{1}(\bar{t})=\dot{\bar{\delta}}_{0}(\bar{t}) \int_{0}^{\bar{t}} \frac{f(\bar{\eta})}{\dot{\bar{\delta}}_{0}^{2}(\bar{\eta})} d \bar{\eta}
$$

where

$$
f(\bar{\eta})=\int_{0}^{\bar{\eta}} \dot{\bar{\delta}}_{0}(\bar{\xi})\left(\frac{d}{d \bar{\xi}} \int_{0}^{\bar{\xi}} \ddot{\bar{\delta}}_{0}(\bar{\tau}) \sum_{j=1}^{n} \frac{m_{0} \phi_{j 0}^{2}}{m_{j}} \cos \bar{\omega}_{j}(\bar{\xi}-\bar{\tau}) d \bar{\tau}\right) d \bar{\xi}
$$

Approximately substituting (84) into $(91), f(\bar{\eta})$ can be simply integrated out as

$$
\begin{aligned}
f(\bar{\eta})= & e_{0}^{4} \sum_{j=1}^{n} \frac{m_{0} \phi_{i 0}^{2}}{m_{j}}\left[\frac{\bar{\omega}_{j}}{\left(e_{0}^{2}-\bar{\omega}_{j}^{2}\right)^{2}}\left(\bar{\omega}_{j} \cos \bar{\omega}_{j} \bar{\eta} \cdot \cos e_{0} \bar{\eta}+e_{0} \sin \bar{\omega}_{j} \bar{\eta} \cdot \sin e_{0} \bar{\eta}\right)\right. \\
& \left.-\frac{\bar{\omega}_{j}^{2}}{\left(e_{0}^{2}-\bar{\omega}_{j}^{2}\right)^{2}}-\frac{\sin ^{2} e_{0} \bar{\eta}}{2\left(e_{0}^{2}-\bar{\omega}_{j}^{2}\right)}\right] .
\end{aligned}
$$

Substituting it into (90) the first order perturbation term is obtained as

$$
\overline{\delta_{1}}(\bar{t})=e_{0}^{3} \sum_{j=1}^{n} \frac{m_{0} \phi_{i 0}^{2}}{m_{j}} \frac{1}{\left(e_{0}^{2}-\bar{\omega}_{j}^{2}\right)^{2}} C_{j}(\bar{t})
$$

where

$$
\begin{aligned}
& C_{j}(\bar{t})=\cos e_{0} \bar{t} \\
& \int_{0}^{\bar{t}} \frac{\bar{\omega}_{j}\left(\bar{\omega}_{j} \cos \bar{\omega}_{j} \bar{\eta} \cdot \cos e_{0} \bar{\eta}+e_{0} \sin \bar{\omega}_{j} \bar{\eta} \cdot \sin e_{0} \bar{\eta}\right)-\frac{e_{0}^{2}-\bar{\omega}_{j}^{2}}{2} \sin ^{2} e_{0} \bar{\eta}-\bar{\omega}_{j}^{2}}{\cos ^{2} e_{0} \bar{\eta}} d \bar{\eta}
\end{aligned}
$$

It can be verified that $C_{j}(\bar{t})$ is a bounded function. So eq. (93) shows that all those terms in the summation whose $\bar{\omega}_{j}$ is much larger than $e_{0} \approx 1.064$ could be neglected because their attribution to the summation are too small to count in. Only those terms whose $\bar{\omega}_{j}$ is not very far from $e_{0}$ have essential effect on the value of $\bar{\delta}_{j}(\bar{t})$. This means that all the effect of the higher modes on the impact can be omitted. Meanwhile, if $\omega_{0}$ is large enough, the lower $j$-th modes $\bar{\omega}_{j}=\omega_{j} / \omega_{0}$ are also very small. In these cases, the effect of lower modes can also be neglected. These conclusions deduced from nonlinear theory are consistent with that of linear theory at the end of section 6 . 


\section{REFERENCES}

[1] W. FLÜGGE, Handbook of Engineering Mechanics, McGraw-Hill Book Company, Inc., 1962.

[2] S. Timosenko And J. N. Goodier, Theory of Elasticity, McGraw-Hill Book Company, Inc, 1951.

[3] W. ZHANG, Impulsive equation by pseduo-velocities and its matrix solution, ACTA Mechanica Solida Sinica, 4 (1983), pp. 520-531. 